\title{
Robotic Strain Imaging for Monitoring Thermal Ablation of Liver
}

\author{
Emad M. Boctor ${ }^{1}$, Gabor Fichtinger ${ }^{1,3}$, Ambert Yeung ${ }^{1,2}$, Michael Awad ${ }^{2}$, \\ Russell H. Taylor ${ }^{1}$, and Michael A. Choti ${ }^{2}$ \\ ${ }^{1}$ Engineering Research Center, Johns Hopkins University, Baltimore, MD, USA \\ ${ }^{2}$ Department of Surgery, Johns Hopkins Hospital, Baltimore, MD, USA \\ ${ }^{3}$ Department of Radiology, Johns Hopkins University, Baltimore, MD, USA \\ eboctor@ieee.org
}

\begin{abstract}
This report describes our initial in vitro results with roboticallyassisted strain imaging for intraoperative monitoring of hepatic tumor thermal ablation. First, we demonstrate a strong correlation between thermal ablation of liver tissue and changes in elastic properties. Second, we present an experimental environment to provide controlled robotically-assisted compression of liver tissue with concurrent ultrasonic reading. Third, we present B-mode strain images of thermally treated liver samples, where the strain images corresponded to the boundaries of histologically confirmed necrosis.
\end{abstract}

\section{Introduction}

Primary and metastatic liver cancer represents a significant source of morbidity and mortality in the United States and worldwide [1]. An increasing interest has been focused on thermal ablative approaches, particularly radiofrequency ablation (RFA). These approaches utilize image guided placement of a probe within the target area within the liver parenchyma. Heat created around the electrode is conducted into the surrounding tissue, causing coagulative necrosis at a temperature between $50^{\circ} \mathrm{C}$ and $100^{\circ} \mathrm{C}$ [2]. Key problems with this approach include localization/targeting of the tumor and monitoring the ablation zone. The first problem has been previously addressed through developing robotic 3DUS system for guidance of liver ablation [3]. The second problem, the subject of this paper, is monitoring the necrosis zone during ablative therapy.

Monitoring the ablation process in order to document adequacy of margins during treatment is a significant problem which often results in either local failure or excessively large zones of liver ablation. Some ablative devices employ temperature monitoring using thermisters built within the ablation probes. However, these temperatures only provide a crude estimate of the zone of ablation. Magnetic resonance imaging (MRI) can monitor temperature changes (MR thermometry), but is expensive, not available in many sites, and difficult to use intraoperatively [4]. Ultrasonography (US) is the most common modality for both target imaging and is also used for ablation monitoring. However, conventional ultrasonographic appearance of ablated tumors only reveal hyperechoic areas from microbubble and outgasing but cannot sufficiently visualize the margin of tissue coagulation. Currently, ablation adequacy is 
only estimated at the time of the procedure and primarily based on the probe position and not the true ablation zone.

The purpose of this study was to assess the ability of US strain imaging to more accurately visualize thermal ablation in the liver. We propose to capitalize on the changes in tissue elastic properties which occur during tissue heating and protein denaturation. It is known that thermal ablation causes significant changes in tissue mechanical properties, including elasticity [5]. Until recently, internal organs such as the liver were not clinically accessible to compression imaging because of the relative resistance of the body wall, and respiratory and cardiac motion artifacts added further difficulties. However, recent trial has been reported performing elasticity imaging of the liver in vivo based on internal cardiovascular motion as a source of compression [6]. Liver tumor ablation is being performed with increasing frequency using operative and laparoscopic approaches. These methods may allow for direct organ compression during therapy, thereby opening the door for more accurate in vivo strain imaging.

\section{Elasticity Imaging}

The main goal of elasticity imaging is remote and non-invasive representation of mechanical properties of tissues. The elastic properties of tissues cannot be measured directly, so a mechanical disturbance must be applied and the resulting response is then evaluated. We can categorize elasticity imaging approaches into static (strain based), dynamic (wave based), and mechanical (stress based) methods [8]. Strain based approach is imaging internal motion under static deformation; dynamic approach is imaging shear wave propagation; and mechanical approach is measuring surface stress distribution. The main components of these approaches: 1) Data capturing during externally or internally applied tissue motion or deformation, 2) Tissue response (displacements, strain, or stress) evaluation, and if needed 3) reconstruction of the elastic modulus based on the theory of elasticity.

The imaging method adopted in this project is ultrasound strain based approach known as elastography, first introduced in 1991 by Ophir et al. [7]. Elastographic imaging techniques have proved that mechanical properties information can be derived from radiofrequency (RF) US images. The objective is to acquire RF US data

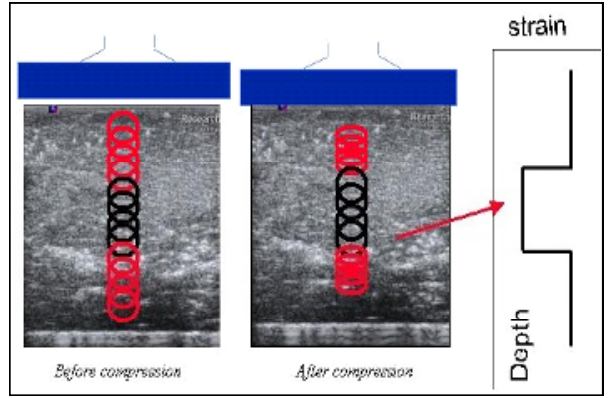

Fig. 1. 2D representation of strain based imaging model. The overlay represents an A-line with 1D cascaded spring system of unequal spring constants. from a tissue in both rested and stressed states, then to estimate the induced strain distribution by tracking speckle motion (Figure 1). The Young's modulus and Poisson's ratio completely describe the elastic properties of an elastic, linear, homogeneous, and isotropic material. Moreover, most soft tissues are incompressible (Poisson's ratio is nearly 0.5) and the Young's modulus can describe the elastic properties. According to Ophir et al. [7], 
larger compressors cause more uniform axial stress fields which allows strain to provide a first order estimate of the Young's modulus. This explains why most elastographic investigations rely on the estimation of the strain distribution.

In the following sections we will focus our discussion on elastography and more specifically, on the signal processing methods used to estimate the strain distribution. Estimating the local strain is therefore an essential step, which requires a high level of accuracy, since the amplitude of tissue deformation is relatively small. So far, the most common signal-processing techniques used in elastography have been gradientbased methods that estimate strain as a derivative from of displacement. These techniques uniformly assume that the medium deformation results in delays between the ultrasound footprints. The echo signal acquired after compression is thus assumed to

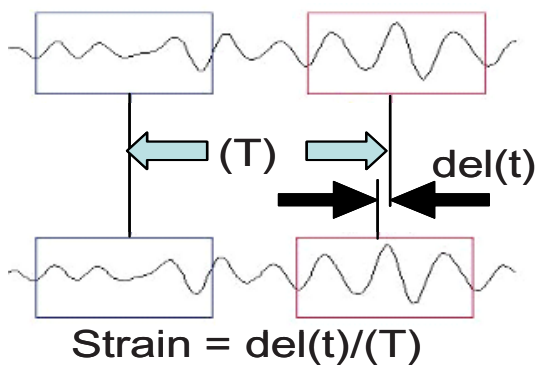

Fig. 2. Illustration of the displacement estimation algorithm applied to an A-line deformation. be a delayed replica of the pre-compression signal. The local tissue displacement is in this case (Figure 2) a simple shift that can be computed as the location of the maximum of the cross-correlation function of gated pre- and post-compression echo signals [8] or as the zero of the phase of the complex correlation function of the corresponding base-band signals [9]. Irrespective to any given Time Delay Estimation (TDE) technique, these methods remain accurate only for very small deformations [0$2 \%$ ] and fail rapidly with increasing strains, because they ignore the signal shape variation induced by the physical compression of the medium. One improvement has been to stretch the entire post-compression signal temporally by an appropriate constant factor prior to time delay estimation [10]. This pre-processing has been demonstrated to compensate fairly well for the effect of compression at low strains. However two fundamental limits arise. First, the value of the proper temporal stretching factor requires an a priori knowledge of the strain magnitude. Second, this factor depends on the local deformation and cannot be constant over the signal. Alam et al. [11] took into account these changes in the shape of signals and considered the signal after compression as a locally delayed and scaled replica of the signal before deformation. The local scaling factor is estimated over each segment of study as the factor that maximizes the correlation between the pre-compression and stretched postcompression signal segments. This study showed that using local scaling factors leads to a method that is more robust in terms of de-correlation noise.

Our implementation, as shown in Figure 2, is based on two techniques. One utilizing the maximization of normalized cross-correlation between pre- and postcompression RF signal after applying 1D companding as described in [10]. The second is more robust and is based on information theoretic delay criterion. Elastography techniques have the potential to be extended into real-time 3D elasticity imaging. The application of pressure field is independent from the imaging array, which provides an opportunity for real-time performance. Other techniques like acoustic radiation force imaging (ARFI) rely on the same array to generate the force pulses (60-70 per line) and to image these responses with 50 tracking beams per pulse [12]. In ARFI, the 
identification of boundary conditions and the extension of the technique to real-time 3D elasticity reconstruction are serious research challenges. It has been known to be of key importance how the compression is actually generated. Freehand elastography is significantly noisier compared to the images from mechanically controlled elastography [14]. Robot-assisted elastography offers several advantages over free-hand approaches: (1) controllable compression to guarantee a strain within the optimal 0.5$2 \%$ range, (2) guaranteed alignment of the direction of strain and the image plane, and (3) relatively straightforward extension to $3 \mathrm{D}$, possibly in real-time. All facts considered, the exploration of robotic assistance in strain imaging appeared to be a logical imperative. In this endeavor, prior expertise of our group in robot-assisted ultrasonography [3] was most useful.

\section{Experimental Design}

The first part of this work was intended to study the variation of liver elastic properties with thermal ablation. A number of researchers have already studied the elastic properties of liver tissues using both healthy and diseased liver tissue excised from experimental animals [13]. However, mechanical elastic testing and modeling of liver tissue undergoing different radio frequency treatment protocols is still lacking. Our study was aimed at measuring the elasticity, i.e. elastic modulus and shear modulus. For these studies, fresh calf liver at room temperature was subjected to focal thermal ablation using RFA (RITA Medical Systems, Inc., Mountainview, CA, XL probe). The liver was cut in $4 \times 4 \times 2 \mathrm{~cm}$ cubes following ablation to testing of tissue elasticity. For these studies, a tensile testing (MTS) machine was used at rate of $30 \mathrm{~mm} / \mathrm{min}$ to produce quasi-static compression up to $20 \%$ strain. Forces were simultaneously recorded both numerically and graphically. In addition, pathological studies were carried out to characterize histologic changes at various stages in the progression of tissue heating. Figure 3 shows the axial compression stress vs. strain curves. These studies demonstrated a trend of increasing slopes and hence increasing Young's modulus values from $20{ }^{\circ} \mathrm{C}$ up to $100{ }^{\circ} \mathrm{C}$. Figure 4 shows the graphical results of the average dynamic shear modulus. The horizontal axis is the frequency of dynamic os-

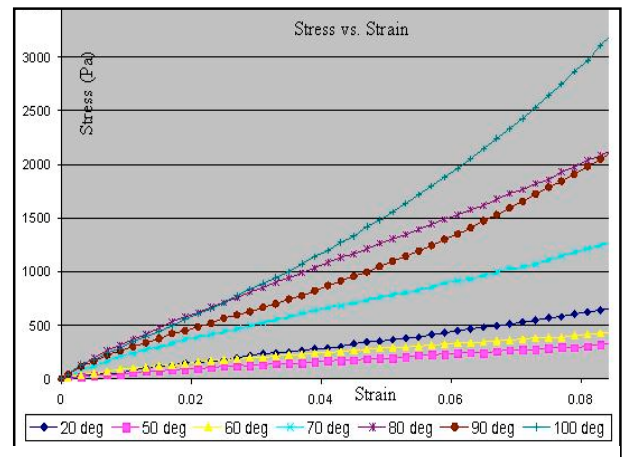

Fig. 3. The axial compression stress vs. strain curves from 20 - 100 degrees C.

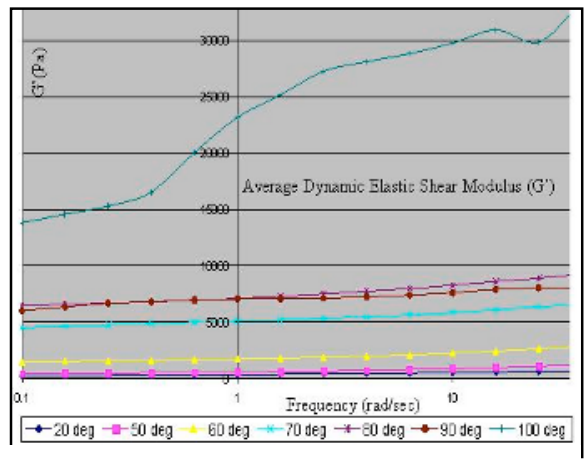

Fig. 4. Average dynamic shear modulus 


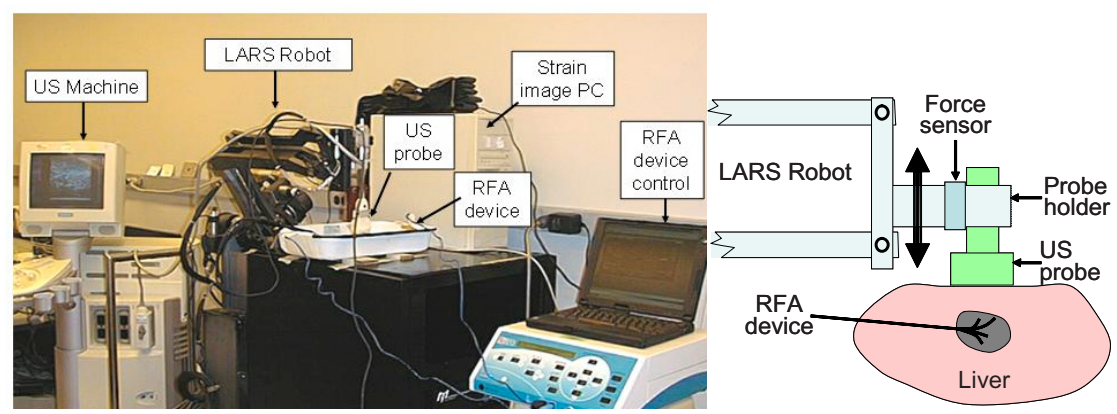

Fig. 5. The overall robotic strain based imaging system (L) and schematic drawing of the robot's end-effector holding the US probe $(\mathrm{R})$. The large probe serves as a compression plate.

cillations in a logarithmic scale. The vertical axis is the dynamic elastic shear modulus (G'). The shear modulus curve also exhibits a positive correlation with temperature. (The details of this elasticity study exceed the limits of this paper.)

The preliminary experiments described above led us to the second phase of this project by designing and implementing a robotic strain imaging acquisition system as shown in Figure 5. We used a Siemens Antares US scanner (Siemens Medical Solutions USA, Inc. Ultrasound Division, Issaquah, WA) with an ultrasound research interface (URI) to access raw RF data. A Siemens VF 10-5 linear array was used to acquire data. The tracking beams were standard B-mode pulses $(6.67 \mathrm{MHz}$ center frequency, F/1.5 focal configuration, apodized, pulse repetition frequency (PRF) of $10.6 \mathrm{KHz}$, with a pulse length of $0.3 \mu \mathrm{s}$ ). The US system utilizes dynamic focusing in receive such that a constant $\mathrm{F} /$ number of 1.5 is maintained. The robot used was a Laparoscopic Assistance Robotic System (LARS) [15]. The LARS is a kinematically redundant manipulator composed of a proximal translation component with a distal remote center of motion component, which provides three rotations and one controlled insertion motion passing through the RCM point. The LARS end-effector, also shown in Figure 5, contained a six degrees-of-freedom force/torque sensor, a translation stage to induce tissue compression with an accuracy of $0.05 \mathrm{~mm}$, and a rotation stage to sweep an US volume. In earlier research, we demonstrated substantial improvements in the 3DUS volume quality, repeatability, planning and targeting with robotic 3DUS vs. freehand scanning [3]. In this project the LARS plays a dual role by helping generate controlled 3D strain data. The thermal ablation system was an RFA generator (RITA Medical Systems, Inc., Mountainview, CA) with XL probe. A Windows 95 laptop recorded real-time temperature readings via thermisters built in the RFA probe. A dual processor workstation interfaced to all system components and capable of controlling LARS to generate 3DUS data with controlled pressure.

\section{Experiments and Results}

Experiments were conducted in triplicate. First, a set of phantom studies were conducted on a gel-based object with a hard spherical inclusion (higher gel concentration) in soft gel background. We have collected 3D strain data by sweeping the robot while 
collecting 2D RF data. Second, a set of experiments were conducted with static monitoring, in which we created $1-2 \mathrm{~cm}$ ablated lesion several hours prior to monitoring. The ablation protocol was heating at $100{ }^{\circ} \mathrm{C}$ for 10 minutes. Although both the phantom and static experiments yielded intriguing results, limitation of space excludes their analysis here.

Third, a set of experiments was based on dynamic monitoring of strain in whole fresh bovine liver, ex vivo. The specimen was soaked in degassed water to remove air pockets, and then was placed in a metal tank to facilitate the grounding for the RFA device (Figure 5.) The protocol of the dynamic study was as follows: (1) System Initialization comprised initializing the robot, Antares in URI mode, the dual processor system, and the ablator device. (We fixed the ablator shaft in a stationary pose with respect to the robot and opened up the tips gradually during the study. (2) Alignment comprised setting the robot arm at 0 degree such that a part of the ablator shaft is always present in the US image. The 90 degree orientation then captures the formed lesion at tip of the RFA device. This way we can study the effect of the ablator shaft and its subsequent shadowing it causes in the strain image. (3) Data collection at room temperature, for reference purposes, at 50, 75, and 100 degrees, for 7 minutes. During acquisition phase the robot moves in multiple compression steps and records force and displacements (boundary conditions) measurements. Displacements have been used to optimize the TDE problem while the force data was not utilized in the calculations. (The force reading will be used at a later stage of the project for the inverse reconstruction problem.)

As we mentioned earlier above, the most decisive component in any elastography processing pipeline is handling time delay estimation (TDE). Advanced TDE algorithms have been implemented by others in the frequency domain, but spectral estimation in case of short signal segments has been an apparently strong weakness of those implementations. R. Moddemeijer searched for a time-domain implementation of a TDE algorithm and defined the so called information theoretic delay criterion [16]. Since this work, however, is still incomplete and no further research has been published toward this direction. In contrast, we used two straightforward TDE estimation algorithms implemented in time-domain, using mutual information and standard normalized cross-correlation. Figures 6 and 7 show consistency of the lesion extracted

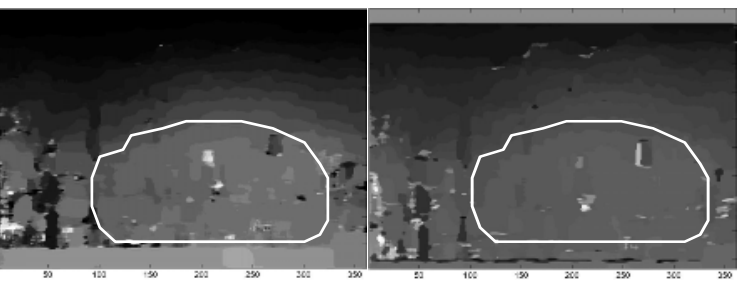
1. Mutual Information Correlation 2. Normalized Cross Correlation

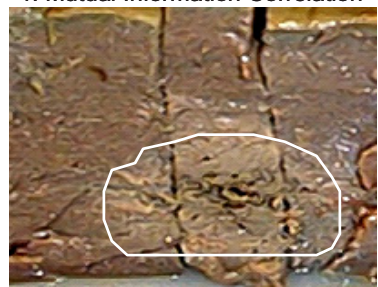

3. Pathological Image

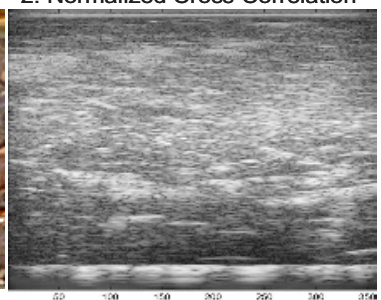

4. B-Mode Ültrasound Image
Fig. 6. Strain images with corresponding pathology and Bmode images at $100^{\circ} \mathrm{C}$, with the RFA device in plane. The white contour is created on the pathological picture and matches with the determined strain images. by these two TDE measures 
and a good matching with the actual pathological images. It is worth noting that the $\mathrm{B}$ mode US images appear to be completely useless in the identification of the ablated region. The perpendicular view in Figure 6 shows decorrelation noise due to the shadowing effects of the needle shaft. In Figure 7, the strain images also show the presence of a large blood vessel that in actual patients would act as a heat sink. The vessel, that represents a small zone of soft structure, caused strongly visible artifacts in the strain images. Also strong correlation can be seen

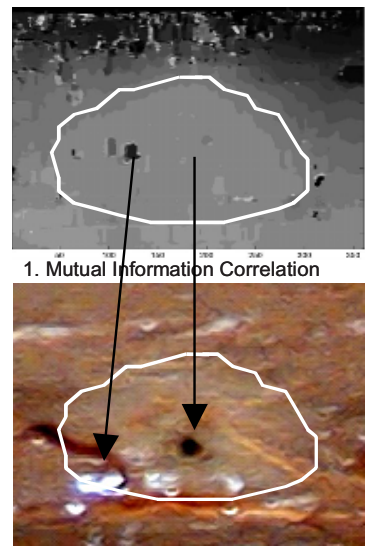

3. Pathological Image

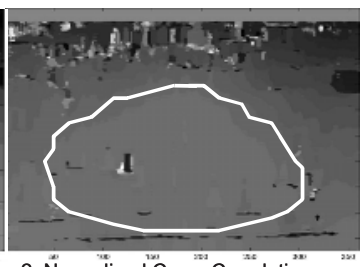

2. Normalized Cross Correlation

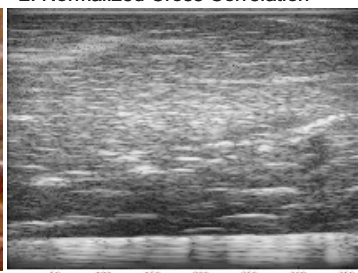

4. B -Mode Ultrasound Image

Fig. 7. Strain images with corresponding pathology and Bmode images at $100^{\circ} \mathrm{C}$, with the RFA device perpendicular to the plane of imaging. The white contour is created on the pathological picture and matches with the determined strain images.

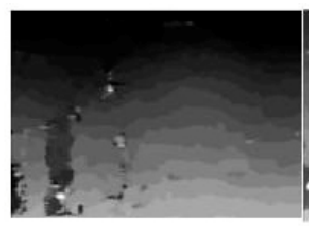

$20^{\circ} \mathrm{C}, 0 \mathrm{deg}$

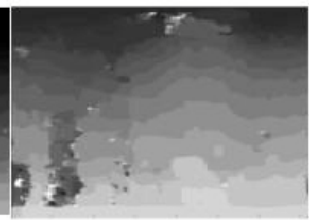

$50^{\circ} \mathrm{C}, 0 \mathrm{deg}$
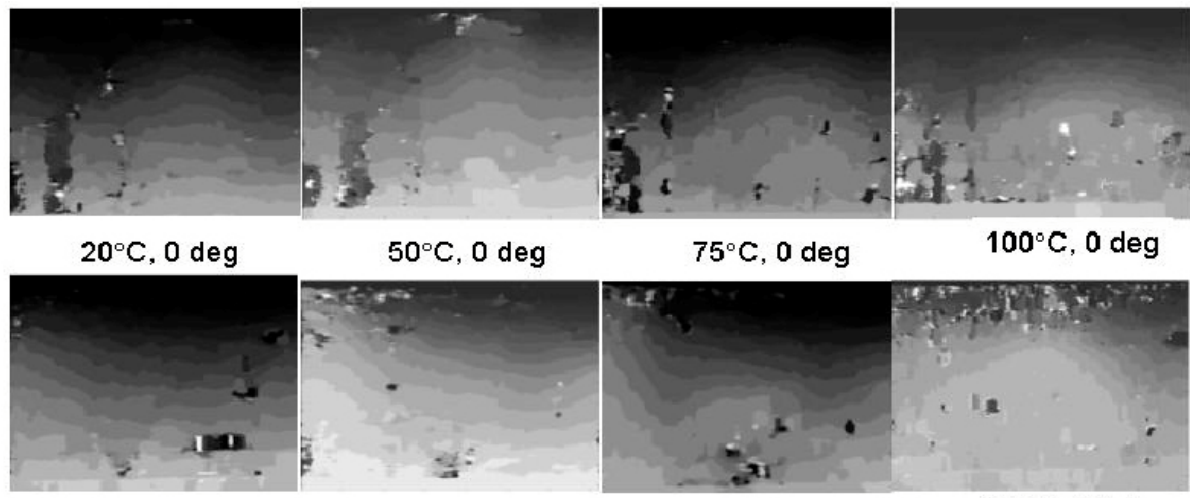

$20^{\circ} \mathrm{C}, 90 \mathrm{deg}$

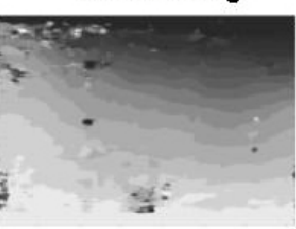

$50^{\circ} \mathrm{C}, 90 \mathrm{deg}$ $75^{\circ} \mathrm{C}, 0$ deg

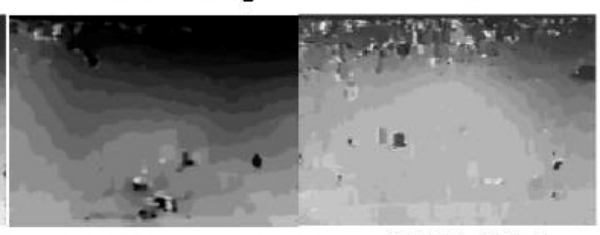

$75^{\circ} \mathrm{C}, 90 \mathrm{deg}$ $100^{\circ} \mathrm{C}, 0 \mathrm{deg}$

$100^{\circ} \mathrm{C}, 90 \mathrm{deg}$

Fig. 8. Series of strain images with mutual information TDE, over several ablation temperatures, in both axial and perpendicular probe positions

between the strain images and the location of the tip of the ablator. Again none of the above discussed fine details could be deduced from the B-mode US images. Figure 8 shows the progression of ablation from room temperature, through $50{ }^{\circ} \mathrm{C}, 75^{\circ} \mathrm{C}$, to $100^{\circ} \mathrm{C}$ ablation temperature, with the use of mutual information TDE. The $20^{\circ} \mathrm{C}$ images show reference strain images that reflect defects due to the presence of the ablator shaft or near by vessel. Moving from $50^{\circ} \mathrm{C}, 75^{\circ} \mathrm{C}$, to $100^{\circ} \mathrm{C}$ we can easily recognize the flower pattern for the Rita device $\left(75^{\circ} \mathrm{C}\right)$. We can also notice the beginning of ablation near to grounding pads (bottom of the images at $50{ }^{\circ} \mathrm{C}$ ).

In summary, robotic strain imaging appears to be promising, while still many particulars of it are under investigation. For TDE, our preference is the mutual informa- 
tion (ME) approach. It needs further optimization for which a strain simulator software framework is being developed.

Acknowledgements. We are grateful to Carol Lowery, Levin Nock, and David Gustafson at Siemens Medical Solutions USA, Inc. Ultrasound Division, Issaquah, WA for providing the Ultrasound Antares unit with the research interface. We also extend our thanks to the JHU Biomechanical Research Laboratory for assistance in tissue compression measurements. This work was supported by NSF EEC 9731478 and an intramural grant from the Johns Hopkins University.

\section{References}

1. Nakakura EK, Choti MA: Management of hepatocellular carcinoma. Oncology (Huntingt). 2000 Jul;14(7):1085-98; discussion 1098-102. Review.

2. Buscarini L, Rossi S: Technology for radiofrequency thermal ablation of liver tumors.Semin Laparosc Surg 1997;4:96-101.

3. Emad M. Boctor, Gregory Fischer, Michael A. Choti, Gabor Fichtinger, Russell H. Taylor: A Dual-Armed Robotic System for Intraoperative Ultrasound Guided Hepatic Ablative Therapy:A Prospective Study. Accepted ICRA 2004.

4. Graham SJ, Stanisz GJ, Kecojevic A, Bronskill MJ, Henkelman RM: Analysis of changes in MRI properties of tissues after heat treatment. Magn Reson Med 1999;42(6):1061-71.

5. Wu T, Felmlee JP, Greenleaf JF, Riederer SJ, Ehman RL: Assessment of thermal tissue ablation with MR elastography. Magn Reson Med 2001 Jan;45(1):80-7.

6. Alexander F. Kolen, Jeffrey C. Bamber, Eltayeb E. Ahmed: Analysis of cardiovascularinduced liver motion for application to elasticity imaging of the liver in vivo. MIUA 2002.

7. Ophir J., Céspedes E.I., Ponnekanti H., Yazdi Y., Li X: Elastography: a quantitative method for imaging the elasticity of biological tissues. Ultrasonic Imag., 13:111-134, 1991

8. Lubinski M.A., Emelianov S.Y., O'Donnell M: Speckle tracking methods for ultrasonic elasticity imaging using short time correlation. IEEE Trans. Ultrason., Ferroelect., Freq., Contr., 46:82-96, 1999.

9. Pesavento A., Perrey C., Krueger M., Ermert H: A Time Efficient and Accurate Strain Estimation Concept for Ultrasonic Elastography Using Iterative Phase Zero Estimation. IEEE Trans. Ultrason., Ferroelect., Freq., Contr., 46(5):1057-1067, 1999.

10. Alam S.K., Ophir J: Reduction of signal decorrelation from mechanical compression of tissues by temporal stretching: applications to elastography. US Med. Biol., 23:95-105, 1997.

11. Alam S.K., Ophir J., Konofagou E.E: An adaptive strain estimator for elastography. IEEE Trans. Ultrason. Ferroelec. Freq. Cont., 45:461-472, 1998.

12. Fahey BJ, Nightingale KR, Wolf P and Trahey GE: ARFI Imaging of Thermal Lesions in Ex Vivo and In Vivo Soft Tissues. Proceedings of the 2003 IEEE US Symposium. 2003.

13. Wen-Chun Yeh, Pai-Chi Li, Yung-Ming Jeng, Hey-Chi Hsu, Po-Ling Kuo, Meng-Lin Li, Pei-Ming Yang and Po Huang Lee: Elastic modulus measurements of human liver and correlation with pathology. US in Med. Biol. 28(4), 467-474, 2002.

14. M.M. Doyley, J.C. Bamber, P.M. Meany, F.G. Fuechsel, N.L. Bush, and N.R. Miller: Reconstructing Young's modulus distributions within soft tissues from freehand elastograms. Acoustical Imaging, volume 25, pp 469-476 2000.

15. Taylor RH, Funda J, Eldridge B, Gruben K, LaRose D, Gomory S, Talamini M, Kavoussi LA, and Anderson JH: A Telerobotic Assistant for Laparoscopic Surgery. IEEE EMBS Magazine Special Issue on Robotics in Surgery. 1995. pp. 279-291

16. Moddemeijer, R., Delay-Estimation with Application to Electroencephalograms in Epilepsy (Phd-thesis), Universiteit Twente, 1989, Enschede (NL), ISBN: 90-9002668-1 